\title{
LEVOMEPROMAZINA E ATROPINA COMO MEDICAÇÕES PRÉ-ANESTÉSICAS NA ANESTESIA PELA ASSOCIAÇÃO TILETAMINA/ZOLAZEPAM, EM CÃES
}

\author{
COMPARISON BETWEEN LEVOMEPROMAZINE AND ATROPINE AS PREMEDICATION \\ AGENTS BEFORE ANESTHESIA USING TILETAMINE/ZOLAZEPAM IN DOGS
}

\author{
Luiz Gonzaga Pompermayer' Flávio Massone ${ }^{2}$ Newton Nunes ${ }^{3}$ Josmari Pirolo $^{4}$
}

\section{RESL 110}

() objetivo desta pesquisa foi avaliar o emprego da atropina e da levomepromazina como me dicaçoes pré-anestésicas para a anestesia pela associaçäo tiletaminaloolazepam. Foram empregados 30 cães, distribuidos em três grupos ignais. O grupo 1 (controle) foi tranado com $0.2 \mathrm{~m} / \mathrm{kg}$ de solução fistológica (placebos por via intravenosa: o grupo $2 \mathrm{com} 0.04 \mathrm{fmg} / \mathrm{hg}$ de sulfato de atropina por via subcutinea e o grupo $3 \mathrm{com} 1 \mathrm{mg} / \mathrm{kg}$ de cloridrato de levomepromazina por via intravenosa. Quinze minutos após, todos os grupos receberam a associação tiletamina/zolazepam na dose de $10 \mathrm{mg} / \mathrm{kg}$ por via intramuscular. Antes da medicação pré-anestésica, 15 minutos após a mesma e aos $15,30$. 60) e 105 mimutos após a administração da associação uletamina/zolazepam foram registrados: $E C G$, temperatura. frequència respiratoria, volume corrente, volume minuto. frequencia cardiaca, pressão arterial, valores hemogasométricos arteriais. graus de analgesia e miorrelaxamento e reflexos protetores. Outros dados como: secreção salivar periodo de latencia periodo anestésico hábil e periodo de recuperação foram igualmente mensurados para efeito comparativo. De acordo com os resultados obtidos concluiu-se que o sulfato de atropina não deve ser administrado cumo medicação pré-anestésica, por potencializar a taquicardia induzida pela assocıação tiletamina/zolazepam. A levomepromazina além de inibir a sialorréia, mantém a estabilidade cardiorrespiratoria e apresenta ação potencializadora dos efeitos anestésicos da associação.

Palavras-chave: anestesia, cães. tiletaminaizolazepam. levomepromazina, atropina

\section{SUMMARY}

\begin{abstract}
The aim of this study was to investigate the effect of levomepromazine and atropine sulfate as a premedication to the dissociative anesthesia produced by a tiletamine/zolazepam combination. Ten dogs were randomly assigned to each of the three groups: control atropine and levomepromazine. Fiffeen minutes before tiletamine/zolazepam. the dogs were treated either with atropine sulfate $(0.044 \mathrm{mg} / \mathrm{kg}$, subcutaneously) or levomepromazine (1.0mg/kg. intravenously), and the control group with saline $(0.2 \mathrm{ml} / \mathrm{kg}$. intravenotusly). The tiletamine/zolazepam combination $(10 \mathrm{mg} / \mathrm{kg}$ ) was administered intramuscularly to all dogs of the three groups. ECG. temperature respiratory rate. tidal volume. minute volume, heart rate, arterial blood pressure, arterial bloodgases, degree of analgesia. sheletal muscle relaxation. ocular. pharyngeal and interdigital reflexes were measured before and 15 minutes after premedication and at 15,30,60 and 105 minutes after tiletamine/zolazepam administration. In addition. the amount of salivary secretion, duration of induction. surgical anesthesia and recovery, were also evaluated. The results suggested that atropine should not be used as premedication in combination with tiletamine/zolazepam due its synergic effect on the tachycardia. Levomepromazine reduced the amount of salivary flow: produced minimal changes in the cardiorespiratory variables and potentiated the anesthesia with tiletamine/zolazepam.
\end{abstract}

Key words: anesthesia dogs, tiletaminelzolazepam. levomepromazine, atropine.

\footnotetext{
${ }^{1}$ Médico Veterinário. Professor do Departamento de Veterinária, Universidade Federal de Viçosa (UFV). 36571-000, Viçosa, MG. Autor para correspondència

${ }^{2}$ Médico Veterinário, Professor da Faculdade de Medicina Veterinária e Zootecnia, UNESP, Campus de Botucatu - Botucatu. SP

${ }^{3}$ Médico Veterinário, Professor da Faculdade de Ciências Agrárias e Veterinárias, UNESP, Campus de Jaboticabal - Jaboticabal - SP.

${ }^{4}$ Médico Veterinário. Professor da Universidade Estadual de Londrina - Londrina - PR. 


\section{INTRODUÇÃO}

A anestesia pela associação tiletamina/zolazepam (T/Z) é pratica, econômica e apresenta ampla margem de segurança, viabilizando sua utilização para uma grande variedade de procedimentos em espécies domésticas e selvagens (LIN $\boldsymbol{e t}$ al., 1993).

Em cães, a associação $T / Z$ na proporção $\mathrm{I}: 1$, promove anestesia suficiente para vários procedimentos porém, a analgesia e o miorrelaxamento não permitem cirurgias abdominais e ortopédicas (FIENI et al., 1988; BENSON, 1989; VEADO, 1992). É efetiva tanto pela via intravenosa (IV) como intramuscular (IM) e a dose é a mesma nos dois casos (SHORT, 1987b). Independentemente da via de administração, verifica-se nesta espécie pronunciada taquicardia (GENEVOIS et al., 1988; TRACY et al., 1988; FIENI et al., 1988; HELLYER et al., 1989; SANDERS et al., 1989; McGRATH, 1989; HOLZCHUH et al., 1990; VEADO, 1992), com diminuição da pressão arterial à indução e retorno aos valores iniciais após 15 minutos (POTOCZAK \& COREY, 1975; HELLYER et al., 1989). Aumento da frequiência respiratória e diminuição do $\mathrm{pH}$ acompanhados de elevação da $\mathrm{PaCO}_{2}$, diminuição da $\mathrm{PaO}_{2} \mathrm{e}$ da saturação da oxiemoglobina foram verificadas por GENEVOIS et al. (1988), também nos momentos iniciais da anestesia.

Como medicação pré-anestésica (MPA) de uso rotineiro, apenas os anticolinérgicos são recomendados, para combater a sialorréia (SHORT, 1987b; FIENI et al., 1988; GENEVOIS et al., 1988; TRACY et al., 1988; HOLZCHUH et al., 1990; VEADO, 1992; LIN et al., 1993) embora, vantagens que incluem maior estabilidade cardíaca, maior miorrelaxamento, maior analgesia e recuperação tranqüila, sejam citadas com o uso de xilazina e ou opióides (SANDERS et al., 1989; BENSON, 1989) e derivados fenotiazínicos (McGRATH, 1989; NATALINI, 1993).

$A$ atropina além de inibir a sialorréia, aumenta a freqüência cardíaca por bloquear as ações do vago sobre o marcapasso nodal sinoatrial (BROWN, 1991), efeito este que, em sinergismo com a taquicardia desencadeada pela associação $T / Z$ poderá resultar em taquicardia severa e risco ao paciente.

Os compostos fenotiazínicos apresentam um amplo espectro de efeitos centrais e periféricos reunindo ação sedativa, simpatolítica, anti-histaminica, antiflogistica, ansiolítica, anti-sialagoga, potencializadora e antiespasmódica (MASSONE, 1994). Dentro deste grupo de medicamentos, a levomepromazina destaca-se por possuir também efeito analgésico
(SOMA, 1971; LUMB \& JONES, 1981; HALL \& CLARKE, 1987). Observações clínicas em cães, indicam que a levomepromazina utilizada como MPA pode reduzir a dose da associação T/Z e impedir a ocorrência de sialorréia, sem apresentar efeitos indesejáveis (NATALINI, 1993).

O estudo comparativo da atropina e da levomepromazina como medicações pré-anestésicas para associação $T / Z$ teve o objetivo de verificar a eficiência da levomepromazina como droga antisialagoga, visando o seu uso em substituição à atropina a fim de obter maior estabilidade cardíaca além da potencialização dos efeitos anestésicos da associação $T / Z$.

\section{MATERIAIS E MÉTODOS}

Foram empregados 30 cães adultos, clinicamente sadios, de ambos os sexos, pesando entre 7 e 19 quilos, separados aleatoriamente em 3 grupos de 10 animais cada.

Todos os animais foram contidos em decúbito dorsal sobre uma calha. $\mathrm{Na}$ área do trígono femoral direito foi infiltrada lidocaína ${ }^{a}$ e a seguir, feita uma incisão da pele de aproximadamente $2 \mathrm{~cm}$ de extensão para visualização e punção da artéria femoral com agulha $13 \times 4,5$ para colheita de amostras de sangue para análise hemogasométrica.

O primeiro grupo (G1) recebeu como MPA, $0,2 \mathrm{ml} / \mathrm{kg}$ de solução fisiológica (placebo), por via IV; o segundo grupo $(\mathrm{G} 2) \quad 0,044 \mathrm{mg} / \mathrm{kg}$ de sulfato de atropina $^{b}$, pela via subcutânea (SC) e o terceiro (G3) recebeu $1 \mathrm{mg} / \mathrm{kg}$ de cloridrato de levomepromazina ${ }^{\circ}$ por via IV. Após 15 minutos, todos os grupos receberam a associação $T / Z^{d}$ na dose de $10 \mathrm{mg} / \mathrm{kg}$ por via $I M$.

As variáveis fisiológicas foram: temperatura retal; freqüência respiratória; freqüência cardíaca; pressão arterial sistólica e diastólica (obtidas através de mensuração indireta ${ }^{\mathrm{e}}$ ); eletrocardiografia ${ }^{\mathrm{f}}$ na derivação II em $50 \mathrm{~mm} / \mathrm{s}$; índice de tono vagal (obtido a partir do logaritmo neperiano da variância dos intervalos R-R, sendo o cálculo feito para cada animal, avaliando-se no mínimo 15 intervalos R-R por momento, conforme SCHWARTZ (1995); volume corrente e volume minuto (obtidos através de ventilometria $\left.{ }^{\mathrm{g}}\right)$ e valores hemogasométricos $\left(\mathrm{pH}, \mathrm{PaCO}_{2}\right.$, $\mathrm{PaO}_{2}$, bicarbonato, $\mathrm{CO}_{2}$ total e excesso de base, dosados em analisador de $\mathrm{pH}$ e gases sanguíneo $\mathrm{s}^{\mathrm{h}}$ ).

As variáveis clínicas foram: analgesia (avaliada de acordo com a resposta apresentada a estímulos dolorosos por pinçamento cutâneo, e classificada conforme os seguintes valores: $0=$ ausente, $1=$ leve, $2=$ moderada, $3=$ intensa); miorrelaxamento 
(avaliado de acordo com o grau de relaxamento da musculatura abdominal e dos membros; classificado da mesma forma descrita para analgesia); reflexos palpebral, corneal, interdigital e laringotraqueal (classificados em presente ou ausente); secreção salivar (com o animal intubado, a cavidade oral foi preenchida com mechas de algodão hidrófilo, provenientes de um frasco hermético previamente pesado. Decorrida uma hora, o algodão foi removido da cavidade, devolvido ao mesmo frasco e novamente pesado em balança de precisão; a diferença de peso, em gramas, foi utilizada para avaliar a quantidade de saliva excretada em uma hora de anestesia); período de latência (tempo compreendido entre a administração da associação $T / Z$ e o momento em que desapareceu o tono da musculatura massetérica e os movimentos de língua, possibilitando a intubação); período anestésico hábil (intervalo de tempo, no qual o animal permaneceu completamente imobilizado sob ação do anestésico dissociativo); período de recuperação (intervalo de tempo entre o primeiro movimento de membros ou cabeça e o momento que o animal adquiriu posição quadrupedal).

Todas as variáveis exceto, secreção salivar e periodos de latência, hábil e de recuperação foram mensuradas nos seguintes momentos: antes da MPA (M1), 15 minutos após a MPA (M2) e aos 15, 30, 60 e 105 minutos após a administração da associação $\mathrm{T} / \mathrm{Z}$ (M3, M4, M5 e M6, respectivamente).

Para o tratamento estatístico dos dados referentes ao grau de miorrelaxamento e de analgesia utilizou-se a prova não paramétrica de Kruskal-Wallis, para amostras independentes. Secreção salivar, período de latência, período anestésico hábil e período de recuperação foram avaliados pela análise de variância para experimento inteiramente aleatorizado, seguida de teste Tukey. As demais variáveis foram avaliadas por meio da análise de perfil. Em todos os casos o grau de significância estabelecido foi de $5 \%(p<0,05)$.

\section{RESULTADOS E DISCUSSÃO}

No G1 e G2 observou-se em todos os animais, reações de dor, quando da administração intramuscular da associação $\mathrm{T} / \mathrm{Z}$, no $\mathrm{G} 3$ essas foram muito discretas. O periodo médio de latência foi de 5,8 minutos no G1; 7,1 no G2 e 4,05 no G3, enquanto o período de recuperação foi de 109 minutos no $\mathrm{G} 1 ; 133$ no G2 e 160 no G3. Resultados esses que não apresentaram diferenças significativas entre grupos. O período anestésico hábil observado no G3 $(88,4$ minutos) revelou-se significativamente superior ao apresentado pelo G1 e G2 (35,5 e 30,5 minutos respectivamente).
Nos 30 minutos seguintes à administração da associação T/Z, o G1 e o G2 apresentaram grau leve de analgesia e de miorrelaxamento. No G3 essas variáveis apresentaram uma graduação significativamente superior, variando de moderada a intensa.

A justificativa de vários autores para a suplementação da anestesia pela associação $T / Z$ com outras drogas, é a obtenção de maior miorrelaxamento e maior analgesia (BENSON, 1989; SANDERS et al., 1989; HOLZCHUH et al., 1990; NATALINI, 1993) e recomendada principalmente para cirurgias que causam grande estímulo doloroso (LIN et al., 1993). No presente experimento, esses benefícios foram evidentes principalmente nos trinta minutos que se seguiram à aplicação da associação $T / Z$, como resultado da administração da levomepromazina no grupo 3 pois, as fenotiazinas entre outras ações, reduzem a atividade motora por deprimir o tronco cerebral e suas conexões com o córtex (MUIR III, 1991). O efeito analgésico da levomepromazina citado por SOMA (1971), LUMB \& JONES (1981) e HALL \& CLARK (1987), não pode de acordo com os resultados deste experimento, ser descartado. Deve-se considerar também que as fenotiazinas elevam o limiar da dor (MASSONE, 1994) e que, embora não se destacando por sua ação analgésica, aumentam a atividade dos fármacos analgésicos (MUIR III \& HUBBELL, 1992).

A quantidade média de saliva apresentada pelo G2 e G3 (2,5 e $1,77 \mathrm{ml}$ respectivamente), foi significativamente inferior à apresentada pelo $\mathrm{Gl}$ $(19,23 \mathrm{ml})$ e indica que tanto a atropina $(\mathrm{G} 2)$ quanto a levomepromazina (G3), foram eficazes no controle da sialorréia. Segundo BALDESSARINI (1991) a redução da salivação pelas fenotiazinas, é entre outras, mais uma manifestação dos seus efeitos anticolinérgi$\cos$. A atropina por sua vez, é o anticolinérgico tradicionalmente utilizado no controle das secreções excessivas desencadeadas por drogas anestésicas (SHORT, 1987a).

A eletrocardiografia revelou arritmia sinusal em todos os animais no M1. Taquicardia sinusal foi observada em $40 \%$ dos animais do G2 após administração da atropina, e em 100\% dos animais do G1 e G2 após administração da associação T/Z. No G3, observou-se ritmo sinusal normal após a associação $T / Z$.

As variações da frequêencia cardíaca (FC), revelaram relação inversa às variações do índice de tono vagal (ITV), ocorrendo aumento significativo da FC após administração da atropina no G2 acompanhado por uma redução correspondente do ITV. Da mesma forma, após a administração da associação $\mathrm{T} / Z$, observou-se elevação da FC, juntamente com 
diminuição do ITV nos três grupos, sendo que, no grupo pré-tratado pela levomepromazina, a elevação da FC foi insignificante, não acarretando taquicardia (Figuras 1 e 2).

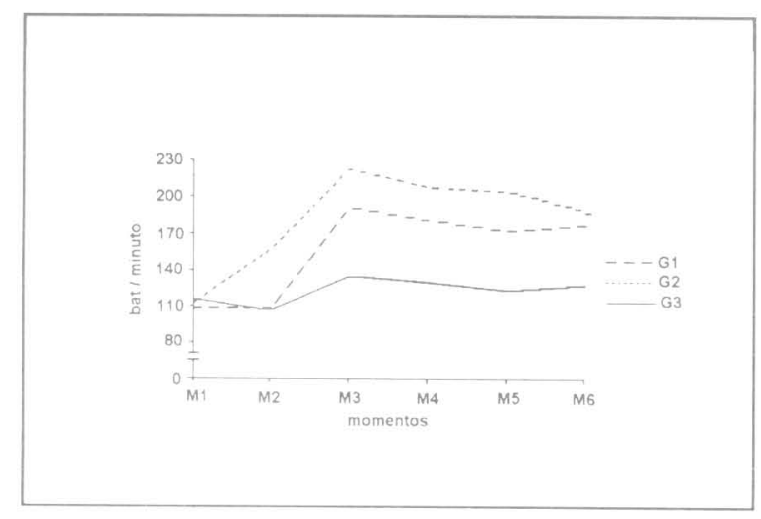

Figura l- Freqüência cardiaca (bat / min.) de càes anestesiados pela tiletamina/zolazepam (G1), tiletamina/zolazepam prétratados pela atropina (G2) ou levomepromazina (G3). em diferentes momentos.

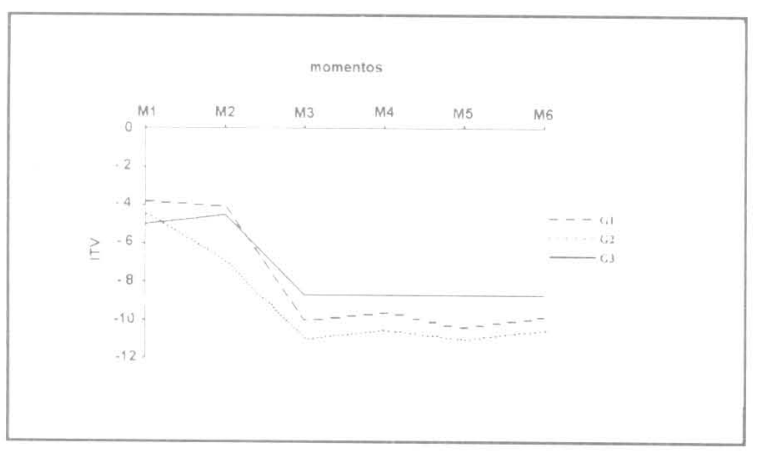

Figura 2- Indice de tono vagal (ITV) de cães anestesiados pela tiletamina (G1). tiletamina/zolazepam pré-tratados pela atropina (G2) ou levomepromazina (G3). em diferentes momentos

A FC do G1 refletiu claramente o efeito taquicardizante da associação T/Z em cães, amplamente enumerado na literatura, sendo devido tanto à ação simpatomimética da tiletamina (CHEN et al., 1969), como à inibição do tono vagal, pois ambas as divisões do sistema nervoso autônomo exercem profunda influência sobre o coração, regulando a FC e a condução atrioventricular (GUYTON, 1992). Em G2, o responsável pela taquicardia, além da tiletamina, certamente foi a atropina que, segundo BROWN (1991) promove bloqueio do efeito vagal sobre o marcapasso nodal SA, o que resultou neste grupo, em sinergismo do efeito taquicardizante. No G3, verificou-se apenas uma elevação não significativa da freqüência cardíaca nos 15 minutos seguintes à administração da associação T/Z, que se manteve até o final do experimento. Para esta estabilidade cardíaca, devese considerar a ação simpatolítica das fenotiazinas que deprimindo central e perifericamente a mobilização de catecolaminas (MUIR III \& HUBBELL, 1992), e bloqueando os receptores $\alpha$-adrenérgicos (GLEED, 1987) reduzem o efeito simpático sobre as fibras cardíacas. O pré-tratamento com levomepromazina propiciou ainda uma menor redução do tono vagal, mantendo neste grupo um ITV significativamente mais elevado que no G1 e G2, o que corrobora as suspeitas de LIN et al. (1993) que, baseados em experimentos com a quetamina, informaram que talvez ocorra bloqueio vagal pela tiletamina, contribuindo para a taquicardia.

A MPA com atropina, é recomendada pelo fabricante da associação $\mathrm{T} / \mathrm{Z}$, com o objetivo de "reduzir o risco de choque vagal, devido à operação". Salvo melhor juízo, esta recomendação pode ser contestada, pois a própria associação anestésica, revelou-se um eficiente bloqueador vagal (Figura 2).

As variações das pressões arteriais sistólica e diastólica, revelaram que dentro de um mesmo grupo não ocorreram diferenças significativas entre momentos. A comparação entre grupos, mostrou que o Gl e o G2 comportaram-se da mesma forma, enquanto o G3 apresentou valores inferiores aos demais grupos com diferenças significativas em M2 e M4 (Figura 3). A maior queda de pressão arterial observada no G3 pode ser explicada pelo pré-tratamento com levomepromazina que como outras fenotiazinas, reduz a pressão arterial em decorrência de uma combinação de ações centrais, bloqueio $\alpha$-adrenérgico periférico e

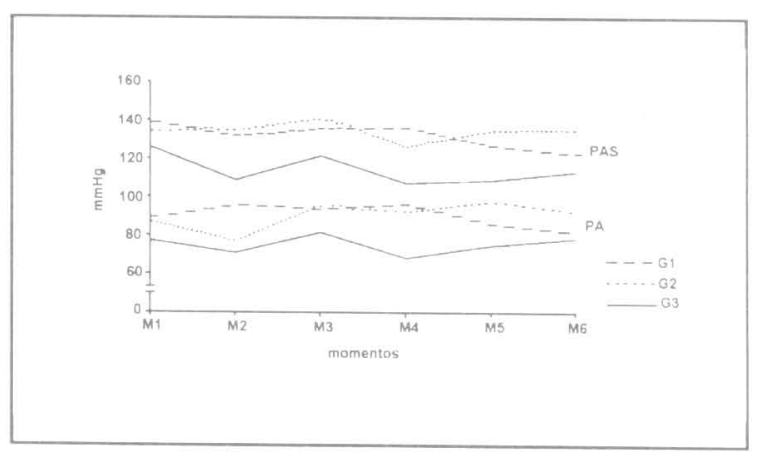

Figura 3- Pressão arterial sistólica (PAS) e diastólica (PAD) de cães anestesiados pela tiletamina/zolazepam (G1). tiletamina/zolazepam pré-tratados pela atropina (G2) ou levomepromazina (G3). em diferentes momentos, 
efeito direto sobre a musculatura lisa dos vasos (GLEED, 1987; BALDESSARINI, 1991; MUIR III, 1991). Embora o efeito hipotensor da levomepromazina tenha se manifestado, em nenhum momento ficou caracterizada hipotensão grave, pois, as médias mais baixas de PAS $(108,3)$ e PAD $(69,1)$ verificadas no G3 em M4, são respectivamente 15 e $11 \%$ inferiores aos valores médios registrados no início do experimento (MI); diferenças essas, que não demonstraram significado estatístico. Assim, a hipotensão causada pela levomepromazina, não pode ser considerada um fator limitante para seu uso como MPA para a associação $\mathrm{T} / \mathrm{Z}$.

A pressão bifásica (queda rápida seguida de elevação), verificada por POTOCZAK \& COREY (1975) e HELLYER et al. (1989) não ficou aqui evidenciada, provavelmente porque neste experimento, os parâmetros foram mensurados após 15 minutos da administração da droga, quando o efeito depressor direto da tiletamina sobre o miocárdio, já havia sido suplantado pela estimulação central com aumento do tono simpático (LIN et al., 1993).

Houve queda significativa da temperatura retal nos três grupos, sendo que no final do tempo experimental o $\mathrm{G} 1$ apresentou redução de $1,02^{\circ} \mathrm{C}$ em relação ao valor inicial, o G2 $1,82^{\circ} \mathrm{C}$ e o G3 $3,17^{\circ} \mathrm{C}$. A comparação dos grupos não mostrou diferença significativa entre o G1 e o G2, enquanto o G3 revelou médias menores que os demais a partir de M4. As fenotiazinas causam depleção das catecolaminas na região do centro termorregulador hipotalâmico e tornam o animal anestesiado, mais suscetivel às mudanças de temperatura do meio ambiente (GLEED, 1987). Os resultados aqui obtidos indicam a necessidade de se prevenir a perda de temperatura, bem como propiciar ambiente adequado no período de recuperação quando um fenotiazínico for utilizado na MPA.

As variáveis respiratórias comportaram-se de forma semelhante nos três grupos, observando-se a partir da administração da associação anestésica (M2), aumento da frequêencia, redução do volume corrente e manutenção do volume minuto, sem diferenças significativas entre grupos. Esta estabilidade foi comprovada pela hemogasometria, quando observouse apenas pequeno declínio do $\mathrm{pH}$ nos três grupos, a partir de 15 minutos após a administração da associação T/Z (M3). Embora no G2 esta diminuição tenha sido significativa nos momentos 4 e 5 , é destituída de valor clínico pois, além de estar dentro dos limites fisiológicos normais, não foram observadas alterações de $\mathrm{PaCO}_{2}$ que indicassem acidose respiratória. $\mathrm{Da}$ mesma forma, o $\mathrm{HCO}_{3}^{-}$, o $\mathrm{CO}_{2} \mathrm{~T}$ e o BE mantiveram-se dentro dos limites fisiológicos. GENEVOIS et al.
(1988) observaram discreta acidose respiratória, cinco minutos após a administração IV de $7 \mathrm{mg} / \mathrm{kg}$ da associação anestésica, o que não foi mais observado após 15 minutos. A não ocorrência de alterações importantes no presente experimento, pode estar relacionada ao fato de que por via IM as alterações tenham sido mais discretas, devido à absorção lenta do produto, ou ainda pode significar que, a rápida regulação pelo organismo conforme relatado por GENEVOIS et al. (1988), tenha ocorrido em um tempo inferior a 15 minutos, que foi $o$ intervalo entre colheitas.

A manutenção dos reflexos protetores, principalmente o corneal e o palpebral, é uma característica da anestesia dissociativa pela tiletamina e zolazepam, enquanto outros reflexos como laringotraqueal e interdigital podem ou não ser suprimidos (SHORT, 1987b). Esta afïrmação, foi comprovada neste experimento, observando-se porém que no G3, tanto o reflexo laringotraqueal como o interdigital estiveram ausentes em um maior número de animais. confirmando mais uma vez, a ação potencializadora da levomepromazina.

Apesar da presença do reflexo laringotraqueal na maioria dos animais, a intubação orotraqueal foi possível após administração da associação $T / Z, 0$ que corrobora as informações de SHORT (1987b). A tentativa de expulsão deixou de existir imediatamente após a sonda ficar em repouso. Nos animais do G3, a intubação revelou-se extremamente fácil devido ao maior relaxamento da musculatura massetérica e da laringe o que permitiu melhor visualização das estruturas de interesse. Outro fator importante foi a ausência do reflexo laringotraqueal em 50\% dos animais deste grupo, bem como sua atenuação nos outros componentes.

Com base nos resultados obtidos neste estudo conclui-se que o efeito anticolinérgico da atropina, deve ser analisado por sua dualidade pois. quando aplicada com o intuito de reduzir o ptialismo causado pela associação T/Z, age sinergicamente com a tiletamina, aumentando ainda mais a FC, enquanto a levomepromazina, além de inibir a salivação, promove maior estabilidade cardiaca e potencializa os efeitos anestésicos da associação, sendo portanto, em cães, a medicação pré anestésica mais vantajosa.

\section{FONTES DE AQUISIÇÃO}

\footnotetext{
a- Xilocaina 1\%- Astra - Brasil

b- Sulfato de Atropina - Hipofarma - Brasil

c- Neozine - Rodia Farma Ltda - Brasil

d-Zoletil (B)50 - Virbac do Brasil - Brasil

e- Cardioscópio Mod. DX 2710 - Dixtal Tecnologia Ind. E Com

Ltda - Brasil

f- ECG 5 - Funbec - Brasil

g- Dräger Volumeter $3000.2000 \mathrm{~K}$ - Drägerwerk $A G$ lüubeck

h- Stat Profile 5 - Nova Biomedical - USA
} 


\section{AGRADECIMENTO}

Os autores agradecem à FAPESP (Fundação de Amparo à Pesquisa do Estado de São Paulo) pelo suporte financeiro e à Faculdade de Medicina Veterinária e Zootecnia da UNESPBotucatu, pela cessão das instalações e equipamentos.

\section{REFERÊNCIAS BIBLIOGRÁFICAS}

BALDESSARINI, R.J. Fármacos e o tratamento de distúrbios psiquiátricos. In: GILMAN, A. G. , RALL, T.W., NIES, A.S. et al. Goodman \& Gilman - As bases farmacológicas da terapêutica. 8. ed. Rio de Janeiro: Guanabara, 1991. Chap. 18, p. 253 287.

BENSON, G.J. Talking about telazol. Vet Med, v. 84, p. 867-874. 1989

BROWN. J.H. Atropina. Escopolamina e drogas antimuscarínicas relacionadas. In: GILMAN, A.G., RALL, T.W., NIES, A.S. et al. Goodman \& Gilman - As bases farmacológicas da terapêtica. 8. ed. Rio de Janeiro: Guanabara, 1991. Chap. 8. p. $98-108$.

CHEN, G., ENSOR. C.R., BOHNER, B. The pharmacology of 2(etylamino)-2-(2-thienyl)-cyclohexanone $-\mathrm{HCl}$ (Cl 634). J Pharmacol Exp Ther, v. 168, p. 171-178, 1969.

FIENI, F., TAINTURIER, D., DENISSEL, E. et al. Uso da associaçăo tiletamina-zolazepam pela via endovenosa na anestesia do cão. Hora Vet, v. 8, p. 45-48. 1988.

GENEVOIS, J.P., AUTEFAGE, A. FAYOLl. A a et al Etude comparée des effets des associations xylazine-kétamine et tilétamine-zolazépam sur quelques grandes fonctions chez le chien. Rec Méd Vet, v. 164, p. 289-296, 1988.

GLEED, R. D. Tranquilizers and Sedatives. In: SHORT, C. E. Principles \& practice of veterinary anesthesia. Baltimore: Williams \& Wilkins. 1987. chap. 3. p.16-27.

GUYTON. A.C. Tratado de fisiologia médica. 7.ed. Rio de Janciro: Guanabara. $1992.830 \mathrm{p}$.

HALL. L.W. CLARKE, K.W. Anestesia veterinária. 8. ed. São Paulo: Manole, 1987. $451 \mathrm{p}$.

HELLYER, P., MUIR HI, W. W., HUBBEli., J. A. E. et al Cardiorespiratory effects of the intravenous administration of tiletamine-zolazepam to dogs. Vet Surg, v. 18, p. 160-165, 1984.

HOLZCHUH, M. P. FIGUEIREDO, A. C. C., CREMONESI, E. Uso da anestesia dissociativa associada a neuroleptoanalgesia em căes. Rev Bras Anestesiol, v. 40, (supl.), p. 106, 1990

LIN, H. C.,THURMON, J.C., BENSON, G.J. et al Telazol - a review of its pharmacology and use in veterinary medicine. $\mathbf{J}$ Vet Pharmacol Ther, v. I6, p. 383-418, 1993.

LUMB, W.V., JONES, E.W. Anestesia veterinaria. México: Continental, 1981.687 p.

MASSONE, F. Anestesiolgia veterinária. Rio de Janeiro: Guanabara, 1994. $252 \mathrm{p}$.

McGRATH, C.J. Talking about telazol. Vet Med. v. 84, p. $867-$ $874,1989$.

MUIR III, W.W. Standing chemical restraint in horses: tranquilizers, sedatives, and analgesics. In: MUIR III, W. W.. HUBBELL, J. A. E. Equine Anesthesia: monitoring and emergency therapy. St. Louis: Mosby, 1991 chap. 11, p. 247-280.

MUIR III, W. W. HUBBEL, J. A. F. Manual de anestesia veterinaria. Zaragoza: Acribia, 1992. Chap.3: Farmacos usados para la medicacion preanestesica: p. 17-31

NATALINI, C.C. Prática clínica anestésica em cães com a associação levomepromazina e tiletamina/zolazepam. Ciência Rural. Santa Maria, v. 23, p. 27-30, 1993.

POTOCZAK, R., COREY, R. The effects of CI-744 upon cardiovascular function in the dog. Fed Proc, v. 34, p. 771, 1975.

SANDERS, E., SHORT, C. E., KEEGAN, R. et al Measuring how dogs respond to Telazol -xylazine combinations. Vet Med, $v$. 84, p. $222-227,1989$.

SCHWARTZ, D. S. Avaliacão eletrocardiográfica, da pressão arterial e do equilibrio ácido-básico em cães submetidos à aplicação tópica do amitraz e tratamento com ioimbina. Botucatu. 1995. 97 p. Dissertação (Mestrado) - Faculdade de Medicina Veterinária e Zootecnia. Universidade Estadual Paulista, 1995

SHORT. C. F. Principles $\&$ practice of veterinary anesthesia Baltimore: Williams \& Wilkins. 1987a. Chap. 2: anticholinergics: p. 8-15.

SHORT. C. E. Principles \& practice of veterinary anesthesia. Baltimore: Williams \& Wilkins. 1987b. Chap. 11: dissociative anesthesia: p. 158-169.

SOMA, L. R. Textbook of veterinary anesthesia. Baltimore Williams \& Wilkins, 1971. Chap. 13: preanesthetic medication p. $121-155$

TRACY, C.H., SHORT, C.E., CLARK, B.C. Comparing the effects of intravenous and intramuscular administration of Telazol. Vet Med, v. 83, p. 104-111, 1988.

VEADO, J.C.C. Estudo comparativo da associação $T / Z$ na anestesia dissociativa em cães. Belo Horizonte, $1992.70 \mathrm{p}$. Dissertação (Mestrado) - Escola de Veterinária. Universidade Federal de Minas Gerais. 1992

Ciência Rural, v. 28, n. 1, 1998. 